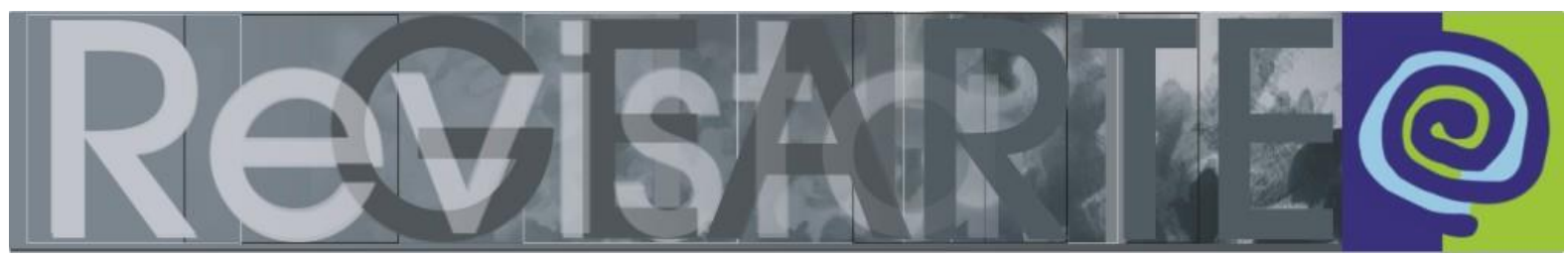

e-ISSN 2357-9854

\title{
Articulação entre a Abordagem Triangular e a Teoria dos Campos Conceituais: propondo diálogos entre a didática e a psicologia cognitiva
}

\author{
Andressa Xavier Zinato de Carvalho (Universidade Federal de São João Del Rei, \\ UFSJ, São João Del Rei/MG, Brasil) \\ Gabriel Dias de Carvalho Junior (Instituto Federal de Minas Gerais, IFMG, Ouro \\ Branco/MG, Brasil)
}

\begin{abstract}
RESUMO - Articulação entre a Abordagem Triangular e a Teoria dos Campos Conceituais: propondo diálogos entre a didática e a psicologia cognitiva - Este trabalho teórico procura realizar uma aproximação entre a Abordagem Triangular, da professora Ana Mae Barbosa, e a Teoria de Campos Conceituais, do psicólogo Gérard Vergnaud. Para tanto, elegemos como foco de nossa análise o sujeito em ação, que é o principal elo entre os dois modelos teóricos. Essa articulação pode ser feita por meio do uso do conceito de "invariante operatório" de Vergnaud, pois ele é capaz de articular as dimensões subjetiva e cultural de uma atividade. A partir daí, encaminhamos o nosso estudo do processo de conceitualização de estudantes em Artes Visuais. Esperamos que este trabalho possa servir de fundamentação teórica para a construção de sequências didáticas apoiadas na ação do sujeito e que, dessa forma, subsidiem tanto professores quanto pesquisadores em Artes Visuais.
\end{abstract}

\section{PALAVRAS-CHAVE}

Abordagem Triangular. Teoria dos Campos Conceituais. Situação. Invariantes operatórios.

ABSTRACT - Articulation between the Triangular Approach and the Conceptual Field Theory: proposing dialogues between didactics and cognitive psychology - This theoretical work seeks to do an approximation between Barbosa's Triangular Approach and the Vergnaud's Conceptual Fields Theory. To do this, we chose as the focus of our analysis the subject in action, which is the main link between the two theoretical models. This articulation can be made through the use of Vergnaud's concept of "operational invariant", since he is able to articulate both subjective and cultural dimensions of a subject in action. We forward our study of the process of conceptualization of students in Visual Arts. We hope that this work can serve as a theoretical basis for the construction of didactic sequences based on the action of the subject and that, in this way, subsidize both teachers and researchers in Visual Arts.

KEYWORDS

Triangular Approach. Conceptual Fields Theory. Situation. Operational invariants.

\section{Introdução}

O ensino de Artes Visuais no Brasil ganhou alguma notoriedade a partir da redemocratização do país, no final dos anos 1980 (BARBOSA, 2008). Com a promulgação da Lei de Diretrizes e Bases da Educação - LDB 9394/96 (BRASIL, 1996) e suas decorrentes diretrizes curriculares, a arte estabeleceu-se como um componente curricular obrigatório. Para que tal componente curricular se consolide, 
de fato, como um espaço de conhecimento e de produção de saberes, deve-se investigar de que maneira os sujeitos constroem os conceitos-chave que dão sustentação para o pensar artístico.

Este trabalho pretende apresentar uma base teórica para que se faça uma articulação entre processos psicológicos ligados ao processo de conceitualização e à dinâmica das intervenções didáticas em espaços formais e não-formais de educação em artes. Nesse sentido, proporemos uma articulação entre a teoria dos campos conceituais (VERGNAUD, 2006) e a Abordagem Triangular (BARBOSA, 2008).

Esperamos, com esse estudo, iniciar um diálogo entre os modelos teóricos citados no sentido de compreender de que maneira, a partir da ação dos sujeitos, os conceitos-chave das Artes Visuais podem ser construídos em atividades que promovam a interação com as obras de arte e entre os aprendizes, considerando o contexto em que se inserem.

\section{Abordagens sobre o Ensino de Artes Visuais: diálogos possíveis entre campos de pesquisas}

\section{A Abordagem Triangular}

Ana Mae Barbosa apresenta um modelo didático para o ensino de artes, chamado de Abordagem Triangular, que, no seu ponto de vista é "[...] construtivista, interacionista, dialogal, multiculturalista e é pós-moderno por tudo isso e por articular arte como expressão e como cultura na sala de aula [...]" (BARBOSA, 2008, p. 337).

Para Barbosa (2014), são três dimensões que devem interagir para que se dê o ensino em artes: o fazer artístico, a leitura de obras de artes (ver) e a contextualização. Essas três dimensões estão representadas na figura a seguir. 
Figura 1 - Representação gráfica das dimensões da Abordagem Triangular de Barbosa.

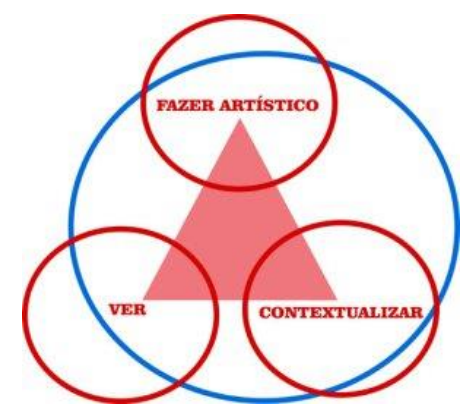

Fonte: Barbosa (2014).

A interpretação dada por Barbosa para a representação acima deve ser compreendida em seu sentido dinâmico, uma vez que, na prática pedagógica, as três dimensões se entrelaçam, sendo mutuamente dependentes umas das outras (BARBOSA, 2014) ${ }^{1}$.

Segundo a autora,

[...] para uma triangulação cognoscente que impulsione a percepção da nossa cultura, da cultura do outro e relativize as normas e valores da cultura de cada um, teríamos que considerar o fazer, a leitura das obras de arte ou do campo do sentido da arte e a contextualização, quer seja histórica, cultural, social, etc. (BARBOSA, 2014, p. 32).

Barbosa (2014) afirma que o contexto assume um papel crucial para o desenvolvimento de situações didáticas em artes que priorizem ação e interpretação dos sujeitos considerando sua linguagem. Devemos sempre lembrar que essa linguagem específica da arte, com seus elementos formais, tem um fomento cultural que permite sua flexibilidade e atualização, dando espaço para o novo que surge no jogo das relações humanas que são permeadas de sensibilidade e criação, traduzidas em ação.

Nesse sentido, uma mesma ação pode ter significados distintos em diferentes contextos, em função da intencionalidade do autor, de seus recursos cognitivos mobilizados e da interação entre ele e o contexto de sua criação. Por exemplo, o traçado de uma linha curva ocorre como elemento da arte barroca ou da abstrata. No

1 Foi feita uma apropriação da Abordagem Triangular nos Parâmetros Curriculares Nacionais, modificando seus componentes para ação, apreciação e reflexão, de $1^{\text {a }}$ a $4^{\text {a }}$ série. $\mathrm{Na}$ concepção da autora, essa modificação não correspondeu às suas ideias para o Ensino de Arte, principalmente no tocante à elaboração de tal documento do qual foi consultora de 5a a 8aㅗ séries (BARBOSA, 2014, p. 31). 
entanto, os sentidos atribuídos a tais linhas, as intenções de seu uso e os resultados obtidos na criação artística e na comunicação com o público são distintos, por se tratarem de contextos de criação e de interpretação diferentes.

Podemos fazer a leitura dessa linguagem a partir de sensações que ela suscita em nós, considerando sua organização num dado momento criativo. Aliás, o que faz da arte um campo do saber tão generalizável está em nós, na nossa qualidade de seres sensíveis e pensantes que captam diferentes organizações estéticas no meio. Damos sentidos a elas por meio de experiências culturais em constantes elaborações e reelaborações pelo nosso cognitivo em concordância com nossa sensibilidade e imaginação.

Contudo, também, pode-se aprender a ler uma obra de arte considerando a sua especificidade, a linguagem nela empregada, os conceitos e elementos pertinentes, o que só enriquece a capacidade do sujeito de perceber a cultura inserida num contexto, desenvolvendo, assim, a sua capacidade crítica e imaginativa em diversas situações do cotidiano, seja ele impregnado de objetos de arte ou não. A possibilidade de perceber a cultura influenciando a linguagem artística e vice-versa é uma abertura que a arte permite sem descaracterizar o seu campo de saber.

Quando estamos diante de um Graffiti², por exemplo, temos a indicação de uma manifestação da cultura estabelecendo mediação com o público a partir de uma linguagem própria. Reconhecido pelo campo de saber da arte, o Graffiti incorpora elementos das histórias em quadrinhos, da publicidade, das imagens televisivas e do cinema em tal contexto artístico.

Ana Mae revela a tendência do ensino de arte com ênfase na contextualização, pois media e impulsiona a prática de professores, dada sua importância para o processo de compreensão nas situações didáticas em artes.

2 As inscrições em muros, paredes e metrôs - palavras e/ou desenhos - sem autoria definida tomam Nova York, no início da década de 1970. Em 1975, a exposição Artist's Space, nessa cidade, confere caráter artístico à parte dessa produção, classificada como graffiti. A palavra, do italiano graffito ou sgraffito, que significa arranhado, rabiscado, é incorporada ao inglês no plural graffiti, para designar uma arte urbana com forte sentido de intervenção na cena pública. Disponível em: <http://enciclopedia.itaucultural.org.br/termo3180/graffiti>. Acesso em: 19 set. 2016. 
Nesse sentido, ela afirma que

[...] a contextualização sendo a condição epistemológica básica de nosso momento histórico, como a maioria dos teóricos em educação comprovam, não poderia ser vista apenas como um dos lados ou um dos vértices do processo de aprendizagem. O fazer arte exige contextualização, a qual é a conscientização do que foi feito, assim como qualquer leitura como processo de significação exige a contextualização para ultrapassar a mera apreensão do objeto (BARBOSA, 2014, p. 33).

No livro chamado Tópicos Utópicos podemos encontrar outra reflexão que busca revisar o antigo termo "História da Arte", tal qual era anteriormente designado na Abordagem Triangular, sendo proposta sua substituição pelo termo "Contextualização", mais abrangente que o primeiro

[...] com o passar do tempo [...], em vez de designar como história da arte um dos componentes da aprendizagem da arte, ampliamos o espectro da experiência nomeando-a contextualização, a qual pode ser história, social, psicológica, antropológica, geográfica, ecológica, biológica, etc., associandose o pensamento não apenas a uma disciplina, mas a um vasto conjunto de saberes disciplinares ou não (BARBOSA, 1998, p. 37-38).

E mais, ainda, sugere a reformulação de sua teoria, dizendo que seria mais interessante representá-la considerando a metáfora do ziguezague e não do triângulo, dado o valor da contextualização tanto para o ver (leitura da obra de arte) quanto para o fazer.

\section{A Teoria dos Campos Conceituais}

A Teoria dos Campos Conceituais começou a ser desenvolvida nos anos 1980 para dar conta da complexidade inerente ao aprendizado das matemáticas, com especial atenção às estruturas aditivas e multiplicativas. Apesar das importantes implicações didáticas, a Teoria dos Campos Conceituais deve ser considerada uma teoria psicológica, visto que seu foco principal é a análise do desenvolvimento do sujeito em ação.

Essa teoria tem sido aplicada em diversos domínios. É possível perceber a interação, entre a Teoria dos Campos Conceituais e as didáticas profissionais (CHAUVIGNÉ; COULET, 2010; GALAND, 2006; LEVAIN; LE BORGNE; SIMARD, 2006). Ela é utilizada no planejamento didático (CARVALHO Jr.; AGUIAR Jr., 2008) e em pesquisas sobre conceitualização em ciências e matemática (ARRIASECQ; 
GRECA, 2006; CUDMANI; PESÁ, 2008; ESCUDERO; JAIME, 2009; MOZZER, 2013; OTERO et al., 2014; PRODANOFF, 2015). Além disso, há um crescente interesse na utilização da Teoria dos Campos Conceituais para investigar a conceitualização em artes (BOURG; GUILLOT, 2015; SOULAS, 2008).

Para sua construção, Vergnaud sintetiza algumas ideias de Vigotski e de Piaget. Da psicologia sócio-histórica, Vergnaud utiliza a noção de Zona de Desenvolvimento Proximal (VIGOTSKI, 2009) e a ideia de que conceitos são ferramentas culturais que podem ser acessadas pelos indivíduos quando da realização de atividades (WELLS, 2008). Da epistemologia piagetiana vieram as ideias centrais sobre a interação sujeito-objeto e a discussão sobre o papel cumprido pelos esquemas em toda a ação do sujeito (PIAGET, 1967).

A Teoria dos Campos Conceituais é orientada para investigar os conceitos durante o seu processo de formação. Por isso, o acento nas situações como aquilo que dá sentido ao conceito.

Para Vergnaud (1991), conceitos funcionam como ferramentas culturais, que comportam três dimensões: as situações (S), as formas de representação $(R)$ e os invariantes operatórios. As situações dão sentido ao conceito, ao prover a base para sua contextualização. É onde os conceitos encontram sua materialidade. As formas de representação simbólica do conceito devem ser partilhadas por determinados grupos sociais para serem frutíferas. Por exemplo, o gesto comumente feito com o polegar levantado indicando ao positivo só é efetivo para representar o conceito de "bom" se, e somente se, o grupo social partilhar essa representação. Já os invariantes operatórios são a parte mais plástica do conceito e possuem duas categorias: os conceitos-em-ação e os teoremas-em-ação. Voltaremos a eles mais à frente.

O processo de conceitualização necessita de uma base subjetiva que torne 0 sujeito capaz de agir. A organização da atividade pelo sujeito é feita por meio de esquemas. Essa noção de esquema, que se iniciou com Kant, foi reinterpretada por Piaget (CARVALHO Jr.; PARRAT-DAYAN, 2015). Vergnaud coloca o esquema no centro da atividade do sujeito. Para isso, ele apresenta que esquemas são orientados para classes específicas de situações. 
Podemos entender os esquemas como sendo a organização invariante da atividade para uma dada classe de situações. Em Vergnaud, esquemas são compostos pelas regras de ação e de antecipação, pelos objetivos e metas, pelos mecanismos próprios de controle e pelos invariantes operatórios.

Os invariantes operatórios são, portanto, o elo entre os conceitos e os esquemas, funcionando como a base conceitual implícita que permite a interação de um sujeito com seu entorno. Esses invariantes operatórios possuem duas classes diferentes e complementares.

A primeira classe representa $\mathrm{o}(\mathrm{s})$ conceito(s) que dado sujeito julga pertinente(s) para abordar determinada situação. Essa admissão de pertinência é feita de forma implícita, motivada pelos dados que o sujeito consegue assimilar da situação enfrentada (CARVALHO Jr., 2013). Esses são os conceitos-em-ação.

Usando os conceitos julgados como pertinentes, o sujeito estabelece proposições com eles no sentido de projetar a sua ação e obter êxito em suas atividades. Essas proposições que o sujeito julga verdadeiras recebem o nome teoremas-em-ação (VERGNAUD, 2011).

Os conceitos-em-ação são, metaforicamente falando, tijolos que servem para construção de proposições que, quando confrontadas com determinado modelo científico, podem ser consideradas falsas ou verdadeiras.

Assim, as interações realizadas entre o sujeito e os objetos ao longo do processo de conceitualização fornecem o cenário para que os conceitos e teoremas em ação possam ser transformados em conceitos científicos. É possível, pois, a partir da investigação dos invariantes operatórios utilizados por determinado sujeito, avaliar como evolui o processo de conceitualização dos sujeitos.

\section{Proposta de Articulação}

A partir do que foi apresentado, a Abordagem Triangular, apesar de ser uma teoria didática que possui um de seus pilares ligados à ação (o fazer), não tematiza os elementos que organizam tal ação dos sujeitos. Nesse sentido, a operacionalidade 
de tal teoria pode ser ampliada para abarcar uma dimensão psicológica ligada à conceitualização.

Assim, parece-nos adequado o diálogo entre a Abordagem Triangular e a Teoria dos Campos Conceituais, uma vez que o conceito de esquema em Vergnaud apresenta uma possibilidade de análise da forma como ocorre o processo de significação em artes que não é explorado pelo modelo de Barbosa.

Em primeiro lugar, no âmbito da Teoria dos Campos Conceituais, é por meio de esquemas que se deve explicar a organização das atividades dos sujeitos. Ao investigar os esquemas utilizados pelos sujeitos durante o processo de conceitualização em artes, pesquisadores da área têm condições de procurar certos modos recorrentes de significação e os conteúdos conceituais que se organizarão em torno de cada ação.

Nesse sentido, quando Barbosa (2008) indica como um dos vértices da Abordagem Triangular a dimensão do $V E R$, os pesquisadores podem analisar essa categoria a partir da investigação dos invariantes operatórios utilizados pelos sujeitos em sua tentativa de interpretar as obras de arte, sejam em ambientes escolares ou não escolares. A cognição em artes nos parece estreitamente ligada à atribuição, em ação, de conceitos pertinentes (cor, perspectiva, traço, pincelada, por exemplo) e o estabelecimento de proposições ("o uso de tonalidades azuladas em uma paisagem reforça a noção de profundidade", por exemplo). A tematização, portanto, dos invariantes operatórios é uma porta de entrada para que se compreendam os mecanismos utilizados pelos sujeitos para construir representações dos fenômenos artísticos.

Esses invariantes operatórios são elementos importantes para que se reconheçam os contextos da ação dos sujeitos. Entendemos que a contextualização proposta pelo professor só se torna operacional quando os estudantes reconhecem, nas situações apresentadas, a possibilidade de utilizar seus esquemas para a realização das atividades. Assim, o elo entre o que a tarefa exige e a organização interna da atividade estabelece-se por meio dos invariantes operatórios. 
Esse é um ponto essencial no planejamento de atividades didáticas. Sabemos que não há construção de novos conhecimentos sem que dado sujeito seja confrontado com situações que exijam a utilização de esquemas ainda não totalmente desenvolvidos. Por outro lado, essas situações não podem exigir que se utilizem esquemas que os estudantes ainda não desenvolveram e estão ainda longe de seu desenvolvimento.

A unidade de análise a seguir, proposta por Carvalho Jr. (2013), é uma tentativa de abarcar essa dinâmica entre conceitos e esquemas.

Figura 2 - Interação esquema-conceito tal como proposta por Carvalho Jr (2013).

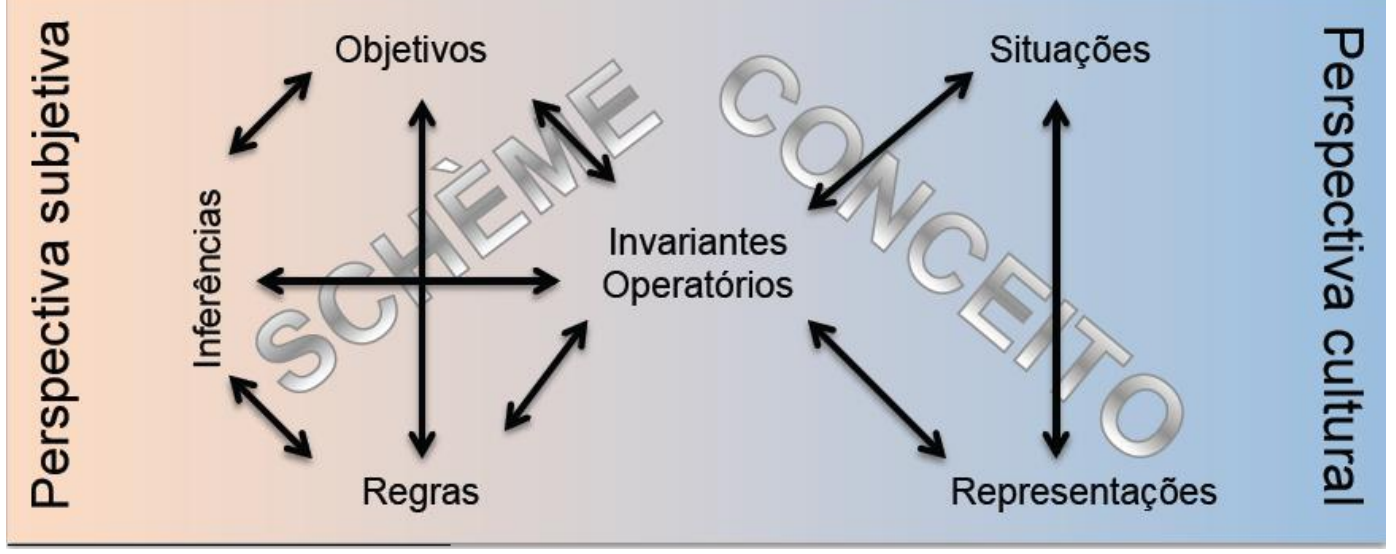

Fonte: Carvalho Jr., 2013.

Nesse sentido, na medida em que são apresentadas situações que desafiem o repertório cognitivo dos sujeitos (seus esquemas já desenvolvidos e aqueles que estão, ainda, em desenvolvimento), promove-se um processo de significação no qual os sujeitos vão se apropriando das ferramentas culturais necessárias para o domínio do campo conceitual das artes visuais, com a possibilidade de criar outras ferramentas, outros esquemas, de melhorar os esquemas já existentes, de diferenciar esquemas ou de assimilar dois esquemas em um mais complexo.

O sujeito em situação, apresentado por Vergnaud, se coloca num fazer contextualizado, sendo essa a premissa para avaliar o seu processo de construção de conceitos dentro de uma determinada área do conhecimento. Avaliar quais conceitos são utilizados pelos sujeitos como criadores no fazer artístico, e também no lugar de leitores de obras de arte, é algo que se dá a partir de um contexto do próprio fazer, da própria obra e de seus desdobramentos culturais. 
Uma situação retirada de uma atividade de pesquisa sobre a formação de cores com 16 crianças da Educação Infantil pode exemplificar o que está sendo dito. Nesta pesquisa, foram utilizadas luminárias de três cores diferentes: azul, verde e vermelha. Cada uma delas era projetada, individualmente, sobre uma tela branca. Logo após, o pesquisador colocava a mão em frente à luminária para fazer a sombra, que era preta. Em seguida, utilizavam-se duas luminárias para se fazer a mistura de cores, obtendose amarelo (verde + vermelho), magenta (azul + vermelho) e ciano (verde + azul). Mais uma vez, o pesquisador colocava a mão na frente. Nesse caso, apareciam três sombras: uma preta e as outras com as cores das luminárias utilizadas. Por fim, as três luminárias eram usadas simultaneamente, para fazer o branco. Colocando a mão na frente, apareciam três sombras (amarela, magenta e ciano) e uma sombra preta central.

Foi solicitado às crianças que, em cada etapa da atividade, explicassem as cores que elas viram na tela e o motivo de as sombras serem coloridas. Em seguida, ao final da atividade, o pesquisador solicitou que fossem realizados desenhos das partes que as crianças julgassem mais interessantes. Todas as 16 crianças presentes desenharam a última parte, em que havia as três luminárias juntas e a mão do pesquisador produzindo três sombras.

Nos desenhos, as crianças utilizaram lápis de cor azuis, verdes e vermelhos e, ao sobreporem essas três cores, obtiveram uma tonalidade bem escura, exatamente o contrário do que elas viram na tela. Elas não reconheceram a contradição entre o que haviam visto e o que haviam desenhado. No entanto, ao serem questionadas sobre isso, a resposta recorrente foi a que cada cor "tampou" a outra, por isso ficou escuro no desenho. Apesar de não dito explicitamente, elas se apoiaram no observável do experimento realizado pelo pesquisador da produção da sombra, no qual a mão, de fato, "tampou" a luz da luminária e, em alguns pontos da tela, ficou escuro. Sem a possibilidade de as crianças desenharem e tentarem explicar o que fizeram, esse modelo, talvez, jamais teria vindo à tona. O conceito-em-ação utilizado foi o de absorção, mesmo que nenhuma das crianças tenha se valido, de forma consciente, dele. 


\section{Conclusão}

Esse trabalho deve ser compreendido como um primeiro passo na tentativa de organização de uma unidade de análise que integre aspectos ligados à didática das Artes Visuais com outros, mais gerais, associados aos processos psicológicos, que nos permitem conhecer e agir sobre os mundos físico e cultural.

Nesse sentido, procuramos, de forma breve, lançar algumas bases para que o diálogo entre a Abordagem Triangular e a Teoria dos Campos Conceituais se torne profícuo e permita tanto a professores quanto a pesquisadores na área se apropriarem das formulações desses dois modelos teóricos. Assim, acreditamos, será possível compreender o sujeito durante o seu processo de significação.

As formulações de Vergnaud e Barbosa respondem a questionamentos/necessidades explicativas diferentes. Nesse sentido, não há identidade perfeita entre os dois autores. No entanto, percebemos alguns pontos de convergência, principalmente no que diz respeito à atenção dedicada à ação dos sujeitos como elemento central da conceitualização.

A organização da sequência de ensino, a partir da ação/do fazer dos estudantes é uma primeira aproximação que julgamos possível da interação entre a Abordagem Triangular e a Teoria dos Campos Conceituais. Nesse sentido, ela representa um passo em direção a uma possível forma de desenho e execução de pesquisas e de intervenções didáticas no campo das Artes Visuais. O foco em um artista que se vale da reciclagem como pano de fundo para sua produção artística revela uma tentativa de trazer um contexto atual e necessário para o maior engajamento das discussões entre os estudantes.

Esse esforço teórico de ler um modelo a partir de outro, verificar pontos de aproximação e de divergência e produzir material didático ainda está em curso e é muito extenso. Torna-se necessário que a sequência didática construída seja aplicada e analisada e que outras sequências sejam produzidas a partir de outros conceitos, outros contextos e outras situações. Assim, será possível avaliar melhor nossas interpretações, inclusive com a comparação dos resultados obtidos de tais 
investigações com os de outras abordagens metodológicas. Sob todos os aspectos, o término deste trabalho é, em essência, um grande começo!

\section{Referências}

ARRIASSECQ, Irene; GRECA, Ileana Maria. A teaching-learning sequence for the Special Relativity Theory at High School level historically and epistemologically contextualized. Science \& Education, n. 21, p. 827-851, 2012.

BARBOSA, Ana Mae. Ensino de arte: memória e história. São Paulo: Perspectiva, 2008.

BARBOSA, Ana Mae. A imagem no ensino de arte. São Paulo: Perspectiva, 2014.

BOURG, Adrien; GUILLOT, Gérald. La didactique de la musique au sein des didactiques disciplinaires: emprunts et comparatismes. Recherche en Éducation Musicale, Québec, p. 43-69, 2015.

BRASIL. Lei n. ${ }^{\circ}$ 9394, de 20 de Dezembro de 1996. Estabelece as diretrizes e bases da educação nacional. Diário Oficial [da] República Federativa do Brasil, Brasília, 20 dez. 1996. Disponível em : <http://www.planalto.gov.br/ccivil_03/leis/L9394.htm>. Acesso em: 11 ago. 2017.

CARVALHO Jr., Gabriel Dias de. Invariantes operatórios na transição entre dois campos conceituais: o caso do tempo relativo. Tese (Doutorado em Educação) - Faculdade de Educação, Universidade Federal de Minas Gerais, Belo Horizonte, 2013.

CARVALHO Jr., Gabriel Dias de; AGUIAR Jr., Orlando Gomes de. Os campos conceituais de Vergnaud como ferramenta para o planejamento didático. Caderno Brasileiro de Ensino de Física, Florianópolis, v. 25, n. 2, p. 207-227, 2008.

CARVALHO Jr., Gabriel Dias de; PARRAT-DAYAN, Silvia. Recortes históricos sobre a noção de schème em Piaget: o processo de desenvolvimento de um conceito. Revista Brasileira de Estudos Pedagógicos, Brasília, v. 96, n. 244, p. 522-540, 2015.

CHAUVIGNÉ, Christian; COULET, Jean-Claude. L'approche par compétences : un nouveau paradigme pour la pédagogie universitaire? Revue Française de Pédagogie, Lyon, p. 15-28, jul-set. 2010.

CUDMANI, Leonor Colombo de; PESA, Marta Azucena. La evolución de los significados de los conceptos científicos en relación con la estructura cognitiva de los estudiantes. Ciência \& Educação, Bauru, v. 14, n. 3, p. 365-80, 2008.

ESCUDERO, Consuelo; JAIME, Eduardo A. Conocimientos-en-acción: un estudio acerca de la integración de las fuerzas y la energía en cuerpo rígido. Investigações em Ensino de Ciências, Porto Alegre, v. 14, n. 1, p. 115-133, 2009.

GALAND, Benoît. La motivation en situation d'apprentissage: les apports de la psychologie de l'éducation. Revue Française de Pédagogie, Lyon, n. 155, abr/jun. 2006.

LEVAIN, Jean-Pierre; LE BORGNE, Philippe; SIMARD, Arnaud. Apprentissage de schémas et résolution de problèmes en SEGPA. Revue Française de Pédagogie, Lyon, p. 95-109, abr./mai. 2006.

MOZZER, Nilmara Braga. O entendimento conceitual do processo de dissolução a partir da elaboração de modelos e sob a perspectiva da teoria dos campos conceituais. Tese (Doutorado em Educação) Faculdade de Educação, Universidade Federal de Minas Gerais, Belo Horizonte, 2013.

OTERO, Maria Rita; et al. La teoría de los campos conceptuales y la conceptualización en el aula de matemática y física. Buenos Aires: Dunken, 2014.

PIAGET, Jean. Biologie et connaissance: Essai sur les relations entre les régulations organiques et les processus cognitifs. Paris: Gallimard, 1967.

PRODANOFF, Fabiana. Enseñanza de nociones básicas de la Teoría Especial de la Relatividad (TER) en la Escuela Secundaria. Tesis (Doctorado en Enseñanza de las Ciencias Mención Física) - Núcleo de Investigación en Educación en Ciencia y Tecnología (NIECyT), Departamento de Formación 
Docente, Facultad de Ciencias Exactas, Universidad Nacional de Centro de la Provincia de Buenos Aires, Tandil, 2015.

RUSSELL, Bertrand. Introduction à la philosophie mathématique. Paris: Payot, 1991.

SÁ, L. A. C. Ensino/aprendizagem da fruição artística: abordagens e métodos. Trabalho de Conclusão de Curso (Licenciatura em Artes Visuais) - Escola de Belas Artes, Universidade Federal de Minas Gerais, Belo Horizonte, 2013.

SOULAS, Brigitte. L'Éducation Musicale: une pratique nécessaire au sein de l'école. Paris: L'Harmattan, 2008.

VERGNAUD, G. La théorie des champs conceptuels. Recherches en Didactique des Mathématiques. Recherches en Didactique des Mathématiques, v. 10, n. 23, p. 133-170, 1991.

VERGNAUD, Gérard. L'explication est-elle autre chose que la conceptualisation? In: LEUTENEGGER, Francia; SAADA-ROBERT, Madelon. (Eds.). Expliquer et comprendre en sciences de l'éducation. Genève: De Boeck, p. 31-44, 2002.

VERGNAUD, Gérard. Au fond de l'action, la conceptualisation. In: BARBIER, Jean-Marie. Savoirs Théoriques et Savoirs d'action. Paris: PUF, p. 275-292, 2011.

VIGOTSKI, Lev. A construção do pensamento e da linguagem. São Paulo: Martins Fontes. 2009.

WELLS, Gordon. Learning to use scientific concepts. Cultural Studies of Science Education, n. 3, p. 329-350, 2008.

\section{Andressa Xavier Zinato de Carvalho}

Possui graduação em Pedagogia pela Pontifícia Universidade Católica de Minas Gerais (2000) e especialização em Gestão da Informação. Atuou como pedagoga da Fundação Centro de Hematologia e Hemoterapia de Minas Gerais. Tem experiência em Educação, atuando principalmente nas seguintes áreas: pedagogia hospitalar, pedagogia empresarial (treinamento e capacitação) e projetos de pesquisa em informática educativa.

E-mail: andressazinato@gmail.com

Currículo: http://lattes.cnpq.br/9833894373385160

\section{Gabriel Dias de Carvalho Junior}

Possui graduação em Licenciatura em Física pela Universidade Federal de Minas Gerais (2000), mestrado em Educação pela Universidade Federal de Minas Gerais (2004) e doutorado em Educação pela Universidade Federal de Minas Gerais (2013). Atualmente é colaborador do Instituto Nacional de Estudos e Pesquisas Educacionais Anísio Teixeira e professor do ensino básico, técnico e tecnológico do Instituto Federal Minas Gerais. Tem experiência na área de Educação, atuando principalmente nos seguintes temas: campos conceituais, desenvolvimento cognitivo, conceito, física e ensino.

E- mail: gabriel.carvalho@ifmg.edu.br

Currículo: http://lattes.cnpq.br/5082825497755276

Recebido em 10 de janeiro de 2017 Aceito em 23 de junho de 2017 